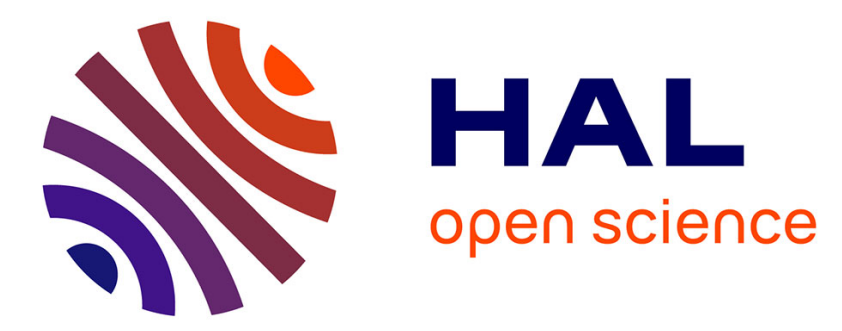

\title{
Generation and Analysis of Chromosomal Contact Maps of Yeast Species.
}

Axel Cournac, Martial Marbouty, Julien Mozziconacci, Romain Koszul

\section{To cite this version:}

Axel Cournac, Martial Marbouty, Julien Mozziconacci, Romain Koszul. Generation and Analysis of Chromosomal Contact Maps of Yeast Species.. Methods in Molecular Biology, 2016, Yeast Functional Genomics, 1361, pp.227-45. 10.1007/978-1-4939-3079-1_13 . pasteur-01419912

\section{HAL Id: pasteur-01419912}

\section{https://hal-pasteur.archives-ouvertes.fr/pasteur-01419912}

Submitted on 16 May 2017

HAL is a multi-disciplinary open access archive for the deposit and dissemination of scientific research documents, whether they are published or not. The documents may come from teaching and research institutions in France or abroad, or from public or private research centers.
L'archive ouverte pluridisciplinaire HAL, est destinée au dépôt et à la diffusion de documents scientifiques de niveau recherche, publiés ou non, émanant des établissements d'enseignement et de recherche français ou étrangers, des laboratoires publics ou privés.

\section{(c)(1)}

Distributed under a Creative Commons Attribution| 4.0 International License 
Generation and analysis of chromosomal contact maps of yeast species

Axel Cournac $^{1,2}$, Martial Marbouty ${ }^{1,2}$, Julien Mozziconacci ${ }^{3}$ and Romain Koszul ${ }^{1,2 \dagger}$

${ }^{1}$ Institut Pasteur, Département Génomes et Génétique, Groupe Régulation Spatiale des

Génomes, 28 rue du Docteur Roux, 75724 Paris Cedex 15, France

${ }^{2}$ CNRS, UMR 3525, 75724 Paris Cedex 15, France

${ }^{3}$ LPTMC, Université Pierre et Marie Curie, 4 place Jussieu, 75005 Paris, France

$\dagger$ Corresponding author. E-mail: romain.koszul@pasteur.fr (R.K.)

Please include a summary and keywords before the introduction. 


\section{Summary}

Genome-wide derivatives of the chromosome conformation capture (3C) technique are now well-established approaches to study the multiscale average organization of chromosomes from bacteria to mammals. However, the experimental parameters of the protocol have to be optimized for different species, and the downstream experimental products (i.e., pair-end sequences) are influenced by these parameters. Here, we describe a complete pipeline to generate 3C-seq libraries and compute chromosomal contact maps of yeast species.

Key words: yeast, chromosome conformation capture, 3C, genome organization, genome assembly, 3C analysis 


\section{Introduction}

We present a method to characterize the tridimensional (3D) organization of budding yeast genomes (see (1), for the first genome-wide analysis performed in S. cerevisiae). Using 3Cseq, a derivative of chromosome conformation capture (3C; $(2-4)$, this protocol generates genome-wide contact maps of various yeast species. The main interest of the $3 \mathrm{C}$-seq approach, compare to other $3 \mathrm{C}$ derivatives such as $\mathrm{Hi}-\mathrm{C}(5,6)$, is that it does not use any enrichment for ligation products and can be directly applied to the sequencing and assembly of unknown species, as described in (7). Briefly, 3 C gives access to the contact frequencies between restriction fragments (RFs) along a chromosome, reflecting the average chromosome organization within the nuclei within a population (2) and, eventually, unveiling functional reorganization upon changes in DNA-related metabolic processes such as DNA repair (8), homolog pairing during meiosis (2) or transcription (9). Interestingly, a related concept was already described in a protocol designed to investigate the organization structure of bacteriophages genomes, through the synthesis and use of a helical DNA-DNA crosslinking agent (10).

Since then, several methods have been published regarding the generation and analysis of $3 \mathrm{C}$ libraries, including a recent comprehensive discussion that recapitulates the overall experimental approach and analysis (11). In its classical version, a cellular culture of a species of interest is treated with a cross-linking agent (typically formaldehyde) that generates covalent bounds between proteins and between DNA and proteins (2). In each cell, cellular components, including the chromosomal set, will "freeze" in a disposition that is assumed to reflect the physiological configuration. To quantify the contacts between 
different DNA regions of the genome, two steps are necessary. First, the cells (and nuclei) are gently lysed and the crosslinked chromatin is digested with a carefully chosen restriction enzyme. The insoluble part of the raw chromatin extract is then isolated through centrifugation, diluted, and incubated in presence of DNA ligase. Using the insoluble fraction diminishes the background by removing small DNA molecules that were not cross-linked in large complexes $(12,13)$. Ligating under diluted conditions aims in turn at alleviating the relegation of molecules which are trapped in the different cross-linked complexes. Following the ligation step, the crosslink is then reversed and the DNA purified. The resulting $3 C$ library consists of a mix of different ligation products whose relative abundance reflects their average spatial proximity within the cell population at the time of the fixation step. The different religation events within a $3 C$ library can be quantified using pair-end (PE) sequencing and genomic contacts maps generated through a variety of protocols $(\mathbf{1}-3,5,14$, 6).

This section describes the experimental protocol for generating and sequencing a $3 \mathrm{C}$ library of a yeast species. The experimental part is then followed by a brief overview of the computational analysis necessary to extract meaningful contact information from the raw data sequencing. Construction and analysis of $3 \mathrm{C}$ libraries do not require special equipment (except obviously access to a sequencing apparatus able to process a large number of PE sequences). However, the preparation of the assay requires careful planning. The choice of the restriction enzyme and of the crosslinking conditions are critical for the success of the experiment, and must be thoughtfully envisioned before starting (Note 1 and Note 2). 


\section{Materials}

\subsection{C Library components}

1. $50 \mathrm{~mL}$ disposable conical tubes.

2. Filtration unit $0.22 \mu \mathrm{m}$.

3. 1.5 and $2 \mathrm{ml}$ lo-binding microcentrifuge tubes (Eppendorf, Hambourg, Germany).

4. VK05 Precellys tube (Bertin Corp, Rockville, Maryland, USA).

5. Yeast species of interest (genome sizes, $\sim 10-15 \mathrm{Mb}$ ).

6. Restriction enzyme and corresponding restriction enzyme buffer (see Note 2).

7. $5 \mathrm{U} / \mu \mathrm{l}$ T4 DNA ligase (Weiss Units).

8. $20 \mathrm{mg} / \mathrm{mL}$ Proteinase $\mathrm{K}$ in water.

9. $10 \mathrm{mg} / \mathrm{ml}$ DNAse-free RNAse A in water.

10. $37 \%$ Formaldehyde solution (v/v) (Sigma-Aldrich, Saint Louis, Missouri, USA).

11. 2.5M Glycine : weigh 75.07g of glycine and transfer to a 1 liter cylinder. Add water to a volume of $400 \mathrm{~mL}$ and dissolve glycine using a magnetic stirrer and a stir bar (see Note 3). Filtrate on a $0.22 \mu \mathrm{m}$ filtering unit and store at room temperature (RT).

12. $10 \%$ Sodium dodecyl sulfate (w/v) (SDS) in water. Add $20 \mathrm{~mL}$ of $20 \%$ SDS (see Note 4) in a $50 \mathrm{~mL}$ disposable conical tube. Add $20 \mathrm{~mL}$ of water. Mix gently by returning tube several times. Store at RT.

13. $20 \%$ Triton $\mathrm{X}-100(\mathrm{v} / \mathrm{v})$ in water. Add $10 \mathrm{~mL}$ of Triton $\mathrm{X}-100$ in a $50 \mathrm{~mL}$ falcon. Add $40 \mathrm{~mL}$ of water and incubate in a $37^{\circ} \mathrm{C}$ water bath until complete dissolution (it can take several hours). Store at RT.

14. $10 \mathrm{X}$ ligation buffer (without ATP): $500 \mathrm{mM}$ Tris $\mathrm{HCl}$ pH 7.4, $100 \mathrm{mM} \mathrm{MgCl}$, $100 \mathrm{mM}$ 
DTT. Add $100 \mathrm{ml}$ of TrisHCl pH 7.5, $20 \mathrm{ml}$ of $\mathrm{MgCl}_{2} 1 \mathrm{M}$ and $10 \mathrm{ml}$ of DTT $2 \mathrm{M}$ to a $500 \mathrm{ml}$ cylinder. Add water to reach $200 \mathrm{ml}$, mix and filtrate on $0.22 \mu \mathrm{m}$ filtering unit. Split as $10 \mathrm{ml}$ aliquot and store at $-20^{\circ} \mathrm{C}$.

15. $10 \mathrm{mg} / \mathrm{ml}$ bovine serum albumin (BSA) in water. Store as $1 \mathrm{~mL}$ aliquots at $-20^{\circ} \mathrm{C}$.

16. $100 \mathrm{mM}$ Adenosine triphosphate (ATP) pH 7.0 in water. Weigh $1 \mathrm{~g}$ of ATP and transfer to a $50 \mathrm{~mL}$ falcon. Add $14 \mathrm{ml}$ of water. Add $1.6 \mathrm{ml}$ of $\mathrm{NaOH} 1 \mathrm{M}$. Complete to $16.7 \mathrm{ml}$ with water. Check that the $\mathrm{pH}$ is around 7.0. Filtrate on $0.22 \mu \mathrm{m}$ filtering unit. Store as $1 \mathrm{~mL}$ aliquots at $-20^{\circ} \mathrm{C}$ (see Note 5$)$.

17. 500 mM EDTA in water, pH8.0.

18. $3 \mathrm{M}$ sodium acetate in water, $\mathrm{pH}$ 5.2. Weigh $204.12 \mathrm{~g}$ of sodium acetate and transfer to a 1 liter cylinder. Complete with water to $400 \mathrm{ml}$, and adjust pH to 5.2 with acid acetic $100 \%$. Complete to $500 \mathrm{ml}$ with water. Filtrate on a $0.22 \mu \mathrm{m}$ filtering unit and store at RT.

19. Isopropanol.

20. 10:9:1 phenol:chloroform:isoamylalcohol pH 8.2.

21. $100 \%$ Ethanol.

22. TE buffer, $\mathrm{pH}$ 8.0. Add $5 \mathrm{~mL}$ of TE $10 \mathrm{X}$ to a $50 \mathrm{ml}$ falcon. Add $45 \mathrm{ml}$ of water and filtrate on a $0.22 \mu \mathrm{m}$ filtering unit. Store at RT.

23. Precellys (Precellys $\left.{ }^{\circledR} 24\right)$ (Bertin Corp, USA) (see Note 6).

24. $16^{\circ} \mathrm{C}$ water bath.

25. $65^{\circ} \mathrm{C}$ oven.

26. Magnetic stirrer and stir bar.

27. Variable temperature incubator $\left(25^{\circ} \mathrm{C}, 30^{\circ} \mathrm{C}\right.$ and $\left.37^{\circ} \mathrm{C}\right)$

28. Dry bath at $65^{\circ} \mathrm{C}$. 
29. Refrigerated tabletopcentrifuge (for $50 \mathrm{ml}$ falcon tubes).

\subsection{NGS Library processing components}

1. Covaris S220 instrument (Covaris Ltd., Woburn, Massachusetts, USA).

2. Snap Cap microTUBE for Covaris (Covaris Ltd.).

3. Column PCR purification Kit (QIAgen, Venlo, Netherlands) (see Note 7).

4. Column MinElute PCR purification Kit (QIAgen) (see Note 7).

5. $1.5 \mathrm{ml}$ lo-binding microcentrifuge tubes (Eppendorf).

6. Illumina paired-end adapters and amplification primers (see Note 8; Illumina, San Diego, California, USA).

7. Tabletopcentrifuge.

8. Nanodrop (Thermo Fisher Scientific).

9. 10X ligation Buffer (New England Biolabs, Ipswich, Massachusetts, USA - NEB) : 500 mM Tris- $\mathrm{HCl}$ (pH 8.0), 100 mM MgCl2, 100 mM DTT, 10 mM ATP.

10. $10 X$ NEBuffer 2 (NEB) : $500 \mathrm{mM} \mathrm{NaCl}, 100 \mathrm{mM}$ Tris- $\mathrm{HCl}(\mathrm{pH} 7.9), 100 \mathrm{mM} \mathrm{MgCl}, 10$ mM DTT.

11. $10 \mathrm{mM}$ Deoxynucleotide triphosphaste in water. Add $100 \mu \mathrm{L}$ of each dNTP (dNTP set $100 \mathrm{mM}$ ) in a microcentrifuge tube $1.5 \mathrm{ml}$. Complete to $1 \mathrm{~mL}$ with water. Make $50 \mu \mathrm{l}$ aliquots and store them at $-20^{\circ} \mathrm{C}$ (see Note 9).

12. $1 \mathrm{mM}$ Deoxyadenosine triphosphate in water. Add $10 \mu \mathrm{L}$ of dATP $100 \mathrm{mM}$ (from the dNTP set) in a microcentrifuge tube $1.5 \mathrm{ml}$. Complete to $1 \mathrm{~mL}$ with water. Make $50 \mu \mathrm{l}$ aliquots and store them $-20^{\circ} \mathrm{C}$ (see Note 9).

13. $10 \mathrm{U} / \mu \mathrm{l} \mathrm{T4}$ polynucleotide kinase.

14. $1 \mathrm{U} / \mu \mathrm{l}$ T4 DNA polymerase .

15. $5 \mathrm{U} / \mu \mathrm{l}$ Klenow DNA polymerase. 
16. $5 \mathrm{U} / \mu \mathrm{L}$ Klenow (exo-) DNA polymerase.

17. $400 \mathrm{U} / \mu \mathrm{I}$ T4 DNA ligase (Cohesive End Unit).

18. Phusion polymerase (Thermo Fisher Scientific).

\subsection{Data processing}

1. Computer with a UNIX system (Linux, MacOSX, Ubuntu). Large memory space and multiprocessor core are needed for efficient reads alignments.

2. Alignment program (for instance, Bowtie2).

\section{http://bowtie-bio.sourceforge.net/bowtie2/index.shtml}

3. A script language like Bash or python to manipulate files.

4. A tool to visualize big matrices like Matlab (license needed) or Octave (free).

\section{Methods}

\subsection{Generation of a 3C library of mixed species}

The generation of the $3 \mathrm{C}$ library takes 3 days, and the generation of the sequencing library an additional 2 to 3 . The $3 \mathrm{C}$ library can be stored at $-80^{\circ} \mathrm{C}$ and therefore the two processes can be separated. Whereas it remains difficult to prepare more than 4 libraries at a time, processing the samples for sequencing can be performed at a larger scale (up to 8 libraries), the limiting step being then, to some extent, the purification of molecules of a size appropriate for sequencing (see Note 10). Therefore, timing is important criteria when planning to do the experiment! The overall schedule will require for an experienced experimentalist an afternoon (partly), a morning - afternoon (partly), a morning (full), 
followed by two full days (with several incubations steps).

\subsubsection{Culture fixation}

1. Start culture of yeast species in your favorite medium. For instance, strains can be grown at $30^{\circ} \mathrm{C}$ in $100 \mathrm{ml} \mathrm{BMW}$ medium (15) up to $1 \times 10^{7}$ cells $/ \mathrm{ml}$ (this quantity will allow to realize two libraries) (see Note 11).

2. Add $8.5 \mathrm{ml}$ of the fresh formaldehyde solution (i.e. $37 \%$ ) to the culture (final concentration of 3\%) (see Note 2).

3. The cells are incubated for 30 minutes at room temperature (RT) under gentle agitation with a magnetic stirrer.

4. Move the cell culture at $4^{\circ} \mathrm{C}$ for another 30 minutes under gentle agitation.

5. Transfer the culture at RT and add $25 \mathrm{~mL}$ of Glycine $2.5 \mathrm{M}$ (final concentration: 470 $\mathrm{mM}$ ) to quench the remaining formaldehyde; incubate under agitation for 5 minutes at RT.

6. Relocate the culture at $4^{\circ} \mathrm{C}$ and keep them under gentle agitation for an extra 15 minutes.

7. Pellet the fixed cells at $4^{\circ} \mathrm{C}(3500 \mathrm{~g}-10$ minutes $)$.

8. Wash the cells with $10 \mathrm{ml}$ of the initial medium.

9. Pellet the fixed cells at $4^{\circ} \mathrm{C}(3500 \mathrm{~g}-10$ minutes $)$.

10. Suspend the cells into $2 \mathrm{ml}$ of medium and transfer them into $2 \times 1.5 \mathrm{ml}$ microcentrifuge tubes.

11. Pellet the cells at $4^{\circ} \mathrm{C}(3500 \mathrm{~g}-10$ minutes $)$.

12. Remove the supernatant and flash freeze the pellet (i.e. in liquid nitrogen or dryice + ethanol). 
13. Store pellets at $-80^{\circ} \mathrm{C}$ until use.

NB: Do not store pellet for more than 6 months (see Note 12)

\subsubsection{C library construction}

Day one

1. Thaw the pellet on ice during 1 hour.

2. Resuspend the cells in $4.5 \mathrm{ml}$ of $1 \mathrm{X}$ restriction buffer (see Note 1 \& 2)

3. Transfer the cell suspension into $3 \times$ VK05 tubes (Precelys) (see Note 6).

4. Lyse the cell using the following program: 9 cycles $\times(6500 \mathrm{rpm}-30 \mathrm{sec}$ ON / $60 \mathrm{sec}$ OFF).

5. Transfer lysate into $8 \times 1.5 \mathrm{ml}$ microcentrifuge tube (500 $\mu \mathrm{l}$ per tube).

6. Add $15 \mu$ l of $10 \%$ SDS per tube (final concentration: $0.3 \%$ ).

7. Incubate tubes in a dry bath at $65^{\circ} \mathrm{C}$ for 20 minutes.

8. Promptly transfer tubes on ice and incubate for 1 minute.

9. Incubate tubes for 30 minutes at $37^{\circ} \mathrm{C}$ and under agitation.

10. Add $50 \mu$ l of triton X-100 $20 \%$ and $6 \mu$ of $10 X$ restriction buffer per tube.

11. Incubate tubes for 30 minutes at $37^{\circ} \mathrm{C}$ and under agitation.

12. Put one tube aside as a non-digested control.

13. Add 150 units of restriction enzyme in each of the 7 remaining tubes.

14. Incubate overnight at the appropriate temperature for the chosen restriction enzyme.

Day two

15. The next morning, take the non-digested control and one of the digested samples 
(non-digested and digested controls, respectively). Add $100 \mu$ l of SDS $10 \%$ and 30

$\mu$ of proteinase $\mathrm{K}$ to each tube and incubate them at $65^{\circ} \mathrm{C}$ overnight (these controls will then be furthered processed at step 30).

16. Centrifuge the 6 remaining tubes at $16,000 \times g$ during 20 minutes at temperature in order to isolate the insoluble fraction of the crosslinked chromatin (12).

17. Remove the supernatant and suspend each pellet in $500 \mu \mathrm{l}$ of $\mathrm{H} 2 \mathrm{O}$.

18. Pool the pellets three by three and dilute the 2 samples in $22.5 \mathrm{ml}$ of a pre-cooled $\left(4^{\circ} \mathrm{C}\right.$ - on ice) ligation reaction mix (10x ligation buffer $2.4 \mathrm{ml}, \mathrm{BSA} 10 \mathrm{mg} / \mathrm{ml} 240 \mu \mathrm{l}$, ATP $100 \mathrm{mM} 240 \mu \mathrm{l}$, water) in $50 \mathrm{ml}$ conical tubes.

19. Add 125 units of T4 DNA ligase.

20. Homogenize the reaction by inverting the tubes 2 to 3 times.

21. Incubate for 4 hours in a $16^{\circ} \mathrm{C}$ waterbath.

22. Transfer to a $25^{\circ} \mathrm{C}$ waterbath for an extra 45 minutes.

23. Add $200 \mu \mathrm{l}$ of EDTA $500 \mathrm{mM}$ per tube to stop the reaction.

24. Add $200 \mu \mathrm{l}$ of proteinase $\mathrm{K}(20 \mathrm{mg} / \mathrm{ml})$ and incubate the tube overnight at $65^{\circ} \mathrm{C}$.

Day three

25. The next morning, cool down the tubes at room temperature and transfer the solution to new $50 \mathrm{ml}$ conical tubes.

26. Add $2.4 \mathrm{ml}$ of $3 \mathrm{M} \mathrm{Na}$ Acetate $\mathrm{pH} 5.0$ and $24 \mathrm{ml}$ isopropanol and incubate at $-80^{\circ} \mathrm{C}$ for 1 hour in order to precipitate DNA (see Note 13).

27. Centrifuge the tube in an appropriate centrifuge at $10,000 \times \mathrm{g}$ during 20 minutes.

28. Remove the supernatant and dry the pellet on the bench (see Note 14).

29. Suspend each pellet in $900 \mu \mathrm{l}$ of TE buffer $1 \mathrm{X}$ and transfer them in $2 \times 2.0 \mathrm{ml}$ 
microtube.

30. Perform a DNA extraction for each tube using $900 \mu \mathrm{l}$ of phenol:chloroform. Also extract the DNA from control samples from step 15 using $500 \mu \mathrm{l}$ of phenol:chloroform:isoamylalcohol.

31. Recover $2 \times 400 \mu \mathrm{l}$ of the aqueous phase (upper phase) for each tube ( $800 \mu \mathrm{l}$ per tube in total) (and $1 \times 400 \mu \mathrm{l}$ for control tubes and transfer them into $1.5 \mathrm{ml}$ microcentrifuge tube.

32. Add $40 \mu \mathrm{l}$ of $3 \mathrm{M}$ Na Acetate $\mathrm{pH} 5.0$ and $1 \mathrm{ml}$ of cold ethanol to each tube.

33. Vortex the tubes and incubate at $-80^{\circ} \mathrm{C}$ for 30 minutes.

34. Centrifuge the tubes at $16,000 \times \mathrm{g}$ for 20 minutes; discard the supernatants.

35. Wash each DNA pellet with $500 \mu$ l of cold $70 \%$ ethanol.

36. Centrifuge tubes at $16,000 \times \mathrm{g}$ for 20 minutes and remove supernatant.

37. Dry pellet by incubating them on a $37^{\circ} \mathrm{C}$ dry bath

38. Suspend each pellet in $30 \mu \mathrm{l}$ TE buffer $1 \mathrm{X}$ supplemented with RNAse A $(0.1 \mu \mathrm{g} / \mathrm{ml}$ final concentration).

39. Incubate at $37^{\circ} \mathrm{C}$ for 45 minutes.

40. Pool the tubes containing the $3 \mathrm{C}$ libraries.

41. Estimation of the quality and quantity on a $1 \%$ agarose gel (Fig. 1; see note 15)

42. Optional: store at $-80^{\circ} \mathrm{C}$ as $\sim 6 \mu \mathrm{g}$ DNA aliquots

\subsubsection{Processing the $3 \mathrm{C}$ library for deep-sequencing}

The current protocol applies if the sequencing apparatus is an Illumina sequencer. For other brands/technologies, refer to the manual to design an appropriate 
protocol.

1. Optional: thaw a dry $3 C$ sample on ice for $x x$ minutes.

2. Adjust the volume of a melted $\sim 6 \mu \mathrm{g}$ aliquot of a $3 \mathrm{C}$ library to $130 \mu \mathrm{l}$ with water. With less DNA the protocol can nevertheless be pursued (down to $x x$ in our experience) but more amplification cycles will be necessary at the end.

3. Shear the library using your favorite instrument. For instance, we use a Covaris with the following settings: Peak Power: 105, Duty Factor 5\%, Cycles per Burst 200, Treatment time (s) 80sec, to obtain DNA fragments between 300 and $1500 \mathrm{bp}$ (see Note 16).

4. Purify the DNA on a QIAquick column and elute with $5 \mu$ of elution buffer (EB).

5. Quantify the DNA on a nanodrop apparatus and prepare a tube with $5 \mu \mathrm{g}$ of DNA and adjust the volume to $80 \mu$ with water.

6. Add $12 \mu$ l of $10 X$ ligase buffer (NEB), $4 \mu$ of dNTP $10 \mathrm{mM}, 15 \mu \mathrm{l}$ of T4 DNA polymerase, $5 \mu$ l of T4 polynucleotide kinase, $1 \mu$ l of klenow DNA polymerase) and complete to $120 \mu \mathrm{l}$ with $\mathrm{H}_{2} \mathrm{O}$. Incubate at RT during 30 minutes.

7. Purify on QIAgen Minelute column and recover $30 \mu \mathrm{l}$ of DNA in EB (to do so, add 31 $\mu \mathrm{l}$ of EB on the column in order to recover $30 \mu \mathrm{l})$.

8. Add $5 \mu \mathrm{l}$ of $10 \mathrm{X}$ buffer NEB2, $10 \mu \mathrm{l}$ of dATP $1 \mathrm{mM}, 3 \mu \mathrm{l}$ of Klenow DNA polymerase (exo minus) and complete to $50 \mu$ with water. Incubate at $37^{\circ} \mathrm{C}$ during 30 minutes followed by 20 minutes at $65^{\circ} \mathrm{C}$.

9. Purify on QIAgen Minelute column and recover $20 \mu \mathrm{l}$ of DNA in EB.

10. Add $3 \mu$ l of $10 \mathrm{X}$ ligation buffer (NEB), $4 \mu$ l of adapters $10 \mu \mathrm{M}$ and $3 \mu \mathrm{l}$ of T4 DNA ligase (NEB). Incubate at room temperature during $2 \mathrm{~h}$ (it is also possible to incubate overnight at $4^{\circ} \mathrm{C}$ ). 
11. Purify fragment between $400 \mathrm{bp}$ and $800 \mathrm{bp}$ with your favorite method (Gel, PippinPrep, caliper - see Note 10). Recover DNA in a volume of $40 \mu \mathrm{l}$ in EB or TE.

12. Determine the optimal number of cycle and quantity of matrix to generate enough library for sequencing. Prepare several PCR reaction (phusion DNA polymerase Volume of $50 \mu \mathrm{L}$ per reaction) with different amount of library (Typically 1 and $2 \mu \mathrm{L}$ ). Temperature profile of the reaction is as follow: $30 \mathrm{sec}$ at $98^{\circ} \mathrm{C}$ followed by 9,12 or $15 \mathrm{cycles}$ of $10 \mathrm{sec}$ at $98^{\circ} \mathrm{C}, 30 \mathrm{sec}$ at $65^{\circ} \mathrm{C}, 30 \mathrm{sec}$ at $72^{\circ} \mathrm{C}$ and a final 7 minute extension at $72^{\circ} \mathrm{C}$.

13. Run the PCR reaction on a $1 \%$ agarose gel and determine the optimal conditions.

14. Prepare 8 PCR reaction using the determine conditions and run them.

15. Purify on 2 QIAquick Minelute columns and recover around $40 \mu$ of DNA.

16. Quantify on Nanodrop and chek the profile on gel.

17. Run your library on an Illumina sequencing platform.

\subsection{Analysis of Pair-End sequencing reads}

Each $3 \mathrm{C}$ based protocol present peculiarities likely to generate noise or specific biases in the data. These caveats can be attenuated by an appropriate, specific preprocessing of the data and by proper normalization. Several approaches have been described that aim at correcting biases, or alleviating it to improve the quality of subsequent analysis (16-18). This part presents the main steps to process the $3 \mathrm{C}$-seq data described above. We provide commands and software's that we currently use. This description is only an illustration of what can be done, since many other bioinformatics tools exist and are available to the community. 


\subsubsection{Mapping along the genomes of the mixed species yeast}

1. If there is a reference genome for the species you are studying, recover or generate the fasta file. If the genome is unknown, see Marbouty et al. (7). For the sake of illustration, we will use in the following the file "genomes_yeasts.fa" as the name of the reference genome.

2. Indexing the reference genome. Aligner software such as Bowtie 2 or BWA are needed for this task and routinely used (19). The first step is to index your reference genome.

> bowtie2 -build genomes_yeasts.fa genomes_yeasts_index

3. Align each mate (from the file sequences_mate1.fastq) independently using the most sensitive mode of the alignment software. We recommend being very stringent when mapping the reads against the genome (whether from mixed samples of from unique samples) to minimize alignment mistakes (Note 18).

> bowtie2 -x genomes_yeasts_index -p6 --sam-no-hd --sam-no-sq --quiet --local -very-sensitive-local -S p1.sam sequences_mate1.fastq

> bowtie2 -x genomes_yeasts_index -p6 --sam-no-hd --sam-no-sq --quiet --local -very-sensitive-local-S p2.sam sequences_mate2.fastq

4. The alignment software generates a SAM file (Sequence Alignment/Map format). You can select and keep the fields relevant for subsequent analysis to save 
memory space using awk. For instance,

$>a w k '\{p r i n t \$ 1, \$ 3, \$ 4, \$ 2, \$ 5 ;\}$ ' p1.sam > p1.sam.select

To recover the pair-end information, a convenient and fast way is to use the bash commands "sort" and "paste":

> sort -T/path_to_temporary_repository p1.sam.select > p1.sam.select.sorted > sort -T/path_to_temporary_repository p2.sam.select > p2.sam.select.sorted > paste p1.sam.select.sorted p2.sam.select.sorted > p1_p2.select.merged

where /path_to_temporary_repository points to a temporary repository where storage space is available for the sort command to be executed.

5. Finally, complete the mapping procedure by filtering ambiguous hits on the genome. Only the pairs of mapped reads with a quality above a certain threshold will be retained (Note 19). $>a w k '\{\$ 5>=40 \& \& \$ 10>=40)$ print $\$ 0 ;\}^{\prime} p 1 \_p 2 \_$merged $>p 1 \_p 2 \_$merged.MQ40 


\subsubsection{Building the contact network}

3.2.3 Assign every pair of mapped read on their restriction fragment by crossing the coordinates of the mapped reads with the restriction map of the genome. This can be done for instance with the "restrict" function from the bioinformatics suite EMBOSS. (Note 20).

\subsubsection{Filtering out of non-informative contacts and construction of the contact map.}

1. A possibility that always arise at the ligation step is the formation of a loop from a long DNA molecule that contains a successive of restriction fragments that are not cut by the restriction enzyme. These events can lead to the detection of false long range contacts and to avoid them, it is necessary to filter out some contacts (17);

Fig. 2). Once this threshold is estimated PE reads that do not present this significant number of RF between them are discarded from the analysis (Note 21).

2. To reduce the dimension of the contact map, and alleviate some local variations resulting from the size of RF, neighboring RF can be pooled into "bins" regrouping the sum of contacts of successive RF. These bins can be made either of a fixed number of successive RF, or in a window constant in size (Note 22).

\subsubsection{Normalization and representation of the contact map}

1. The raw matrix is then normalized to attenuate biases inherent to the protocol

(Note 23). Several methods can be used to achieve this step, including the Sequential Component Normalization (SCN; (16); Note 24). 
For instance, using MatLab (Mathworks, Natick, MA, USA), the contact map Mat is normalized through several iterations of the following commands:

$$
\begin{aligned}
& >\text { for } i=1: 1: n 1 \\
& >\text { Mat2(:,i)=Mat }(:, i) / \text { norm }(\operatorname{Mat}(:, i)) ; \\
& >\text { End } \\
& >\text { for } i=1: 1: n 1 \\
& >\text { Mat_scn }(i,:)=\operatorname{Mat2}(i,:) / \text { norm }(\operatorname{Mat} 2(i,:)) ; \\
& >\text { end }
\end{aligned}
$$

2. The correlation contact map of the normalized matrix can also be computed (Note 25; Fig. 3). Using Matlab, the corrcoef function calculates the Pearson correlation coefficient between each line and column of the normalized map.

> Mat_corr=corrcoef(Mat_scn);

3. To visualize the contact map, the figure function from Matlab is a convenient tool. The visualization can be improved by multiplying the matrix with exponential coefficient to increase contrasts (Note 26) > figure, imagesc(mat.^0.4);

\subsubsection{Statistical analysis}

1. Although genomic contact maps unveil the global genome organization of a population of cells, such as centromere clustering that reveal the position of 
centromeric sequences in yeast species $(1,4)$, a large fraction of the information contained in the data is not directly visible on the contact map and need to be statistically exploited. The statistical analysis aims at determining whether the contacts observed between two or more DNA regions of interests is higher than expected by chance. One way to do that is to calculate the mean of normalized interactions between the different members of the group of interest and compare them to a random set to evaluate significance (Note 27).This implies carefully designing a null model taking into account the specificity of the global chromosome organization. 


\section{Notes}

1. The choice of the restriction enzyme and buffer is a key component of this experiment. Several restriction enzymes become inactive under the experimental conditions described in the protocol (i.e. cellular brut extract of yeasts). The cheapest enzymes - usually the best characterized - provide the best candidates to generate a 3 C library. Consequently, we highly recommend choosing " classical » restriction enzymes when designing the experiment. However, it is still possible that the enzyme selected is not active enough (for instance Sacll works pretty bad in our hands; MM, personal communication). Restriction buffer used to construct $3 \mathrm{C}$ libraries have to contain DTT. Consequently, we strongly suggest avoiding new NEB buffer (NEBuffer 1.1, 2.1, 3.1 and CutSmart)

2. Building a $3 \mathrm{C}$ library is also entirely dependent on the match between the restriction enzyme chosen and the condition of the fixation step. The likelihood for a RF to be crosslinked is dependent on the probability for one bp to be crosslinked and thus on the incubation parameters in the presence of a fixative agent level (17). Notably, a 4-cutter (restriction enzyme recognizing a 4pb site) will require a higher concentration of crosslinking agent (or longer incubation, to some extent) than a 6cutter. The protocol described in this article is designed for enzymes that generate RFs with an distribution average lower than $500 \mathrm{bp}$ (+/- $200 \mathrm{bp})$. In general, we do not recommend enzymes that generate RF with an distribution average lower than $300 \mathrm{bp}$.

3. Dissolving glycine at such concentration can take several hours. The process can be accelerated by gently warming the solution $\left(40-50^{\circ} \mathrm{C}\right)$.

4. SDS treatment is a critical step. We noticed an important drop in the quality of the 
library when the SDS begins to precipitate (warming prior use does not solve the problem). Therefore the SDS solution has to be changed immediately is signs of precipitation are visible.

5. ATP is a critical cofactor of the ligase reaction. In order to avoid any problem due to ATP degradation, discard the thaw aliquot after use.

6. Some yeast species - or some metabolic states - appear somehow resistant to zymolyaze treatment. Lysis is thus obtained through mechanical treatment. Other solutions than Precellys (Bertin Corp, Rockville, Maryland, USA) can, of course, be envisioned.

7. We have noticed a decrease in the efficiency of the QIAgen PE buffer (i.e. wash buffer) over time. To avoid this problem we strongly recommend preparing the required amounts extemporarily to the experiment.

8. Sequences of Pair-End Adapters (with index) and Pair-End Amplification Primers can be found at http://support.illumina.com/downloads/illumina-customersequence-letter.html

9. As for ATP, discard aliquot once thaw and used.

10. For size selection, we routinely use a Pippinprep apparatus (Sage Science), though gel purification works well.

11. The mixed culture of $100 \mathrm{ml}$ with a concentration of $1 \times 10^{7}$ cells of genome sizes $\sim 10-15 \mathrm{Mb}$ is sufficient to generate 2 libraries in the conditions described in the protocol. For other conditions, the crosslinking step will have to be adapted, as it will change the ratio DNA-Protein-formaldehyde (see Notes 1 and 2).

12. We have noticed a quality decreased after storage of more than 6 months.

13. After 1 hour at $-80^{\circ} \mathrm{C}$, solution will froze. Prompt freezing is necessary for a good 
recovery of libraries.

14. The pellet do not have to be entirely dry, since DNA will be subsequently extracted by a phenol:chloroform step and re-precipitated.

15. Quantify the libraries on a gel using an image quantification software (such as Image J or Quantity One). Indeed, large DNA fragments and impurities prevent use of Nanodrop or Qbit quantification.

16. Shearing can also be done using Bioruptor or nebulizer.

17. If you make an experiment with several organisms in the same culture, index all the genomes in once (7). Doing that, identical or very similar sequences between several organisms could be identified in the alignment process.

18. Importantly, the paired-end mode of the software Bowtie2 must not be used to align $3 \mathrm{C}$ or $\mathrm{Hi}-\mathrm{C}$ data. Indeed, this mode sometimes favors wrong positions for ambiguous reads. Notably, it can favor a cis position for two ambiguous PE reads against a distant or trans alignment. This leads to "speckles" in trans positions between the regions involved, which often correspond to repeated sequences (for example rDNA genes) and can generate mistakes when analyzing co-localization of DNA regions.

19. Based on our experience, the Mapping quality threshold must be set as high as possible. Generally use a quality value of 40 . Even then, incorrect mapping can still be detected (for example, we still have reads with correct mapping quality thus not filtered that are mapped on $\mathrm{Y}$ chromosome in female human cells). Another possibility to filter ambiguous reads is to keep the reads that do present a 'XS' field in the SAM file. The 'XS' option contains the score of the second best alignment and therefore is an indicator of a non-unique alignment. This approach is used in 
the python library hiclib (18). However, in our hands, this approach is relatively less stringent than putting a threshold on the Mapping Quality.

20. A typical 3C-seq library contains a large amount of molecules that do not result from religation of two non-adjacent $\mathrm{RF}$, and therefore that do not bring information about the 3D genome structure and have to be removed. To do so, plot the distribution of events according to the orientation of the pair-end reads compare to the reference genome coordinates. We distinguish at least 3 categories (17). First, " uncut » events that correspond to mate pairs separated by none, or a few consecutive RF, most likely not digested. "Loops", that correspond mostly to one or several consecutive RF circularized during the ligation step and subsequently sheared. Finally, "weird" events which are pairs of reads belonging to the same restriction fragment but with same directions of reading along the reference genome. This type of events can be explained by the presence of several copies of the same fragment in the ligation (due to rare religation between $\mathrm{RF}$ from different cells, replication, or diploid cells).

21. We assign each read to its restriction fragment along the genome using a custommade $\mathrm{C}$ routine. The $\mathrm{C}$ language is fast and allows precise allocation of memory. At this step, the distance between each read and the associated restriction site and keeps as well the size of the associated restriction fragment can also be calculated. Several other filters can be applied at this stage to remove incorrect events. Notably, the size of the fragment sent to the sequencer can also be calculated and the distribution can be checked to be in accordance with the experimental one (see step 11 selection with PippinPrep). A filter can also be applied to reads too close of their associated restriction site. 
22. The "visibility" of RF in the PE reads will depend principally of their size, and whether they contain repeated sequences or not. This variability will impact on the visibility of the bins. Therefore, working with fixed size bins may reflect this variation in visibility. There is no perfect solution, since working with bins made of successive RF (and therefore representing regions of different sizes) can also generate visual discrepancies between bins: for instance, a bin made of a successive long RF will be represented in the contact map the same way as a bin made of successive small RF. Working with fixed size bins can also simplify the analysis and comparison between contact maps. In this case, the genome is divided in equal size bins and every reads is attributed to the bin where its start position belongs.

23. The theory behind the normalization procedure is that each bin has an equal probability to be detected overall. Plotting the distribution of contact per bin reveal a subset of elements that are undetectable or present only a handful of contacts. These bins can either result from repeated sequences that prevent confident mapping of reads in these regions, but potential structural variations between the genome of the strain being tested and the reference genome can also generate a similar outcome (for instance, deletion can easily be detected with such approaches, since consecutive bins will present no contacts; 20 ). The tail of this distribution has to be removed since these bins correspond clearly to "invisible" regions. The remaining graph presents a clear Gaussian distribution, meaning that some bins are less detectable than others. Such differences result notably from variations in the distribution of RS if the bins in the contact map are made of constant sizes, or from differences in the sizes of RF binned together if this binning 
approach has been retained. To attenuate the differences of detection, divide each matrice element by the sum of elements of the line it belongs then do the same by dividing each matrice element by the sum of the column it belongs. Iterate this process until the matrice converges to a stable one. To our experience, a few iterations $(5-10)$ is sufficient to have a stable matrice. The normalization procedure ensures that the sum over the column and lines of the matrix equals 1 , which reduces the noise and biases inherent to the protocol. You can do that in a matlab script, C code or python code.

24. The SCN procedure is based on similar approaches and mathematical operations than the ICE procedure developed by the Mirny lab (18). To our knowledge, it gives similar results.

25. Each element of the correlation matrix corresponds to the Pearson coefficient between the line and column vectors. Correlation map are to be handled with care, since they do not reflect necessary important contacts between two elements, but provide indications about their behavior similarity. This representation will increase the contrast between elements presenting similar neighbors, and others positions, but will not provide indications on the strength of the contacts between these elements.

26. "Beautification" of the contact map can be increased by applying a blurring effect on the matrices. Applying a convolution matrix with as kernel the $3 \times 3$ matrix $[0.05$ $0.050 .05 ; 0.050 .050 .05 ; 0.050 .050 .05]$ to the contact map will result in such effect. The convolution has to be repeated to emphasize the structures. You can as well display the matrices with $\mathrm{R}$ that contains several gaussian or in python with the tool imshow which contains an interpolation function as default. It has to be 
noted that this type of image processing adds information to the initial data, and that statistical analysis cannot be done with such processed matrixes.

27. Choosing a relevant null model is not trivial. The minimalist null model must respect the distribution along the different chromosomes as proposed in (21). A more stringent null model has to take into account the global organizational features of the genome being studied. For yeasts, whose chromosomes are organized under a Rabl organization with centromeres co-localizing and chromosome arms extending from there in the nuclear space, the positions along the genome of the elements being studied has to take into account their distance from the centromeres and, eventually, from the subtelomeric regions. Otherwise, if a subset of genes appear to be positioned at equal distances from their respective centromeres they will mechanically present enriched contacts due to the constraint imposed by centromere clustering. For instance, studying colocalization of coregulated, paralogous genes in S. cerevisiae raises this question accurately, since many of those genes originated from whole genome duplication events and have remained at relatively equal distances from centromeres. Failing to take into account this disposition in the null model will lead to the conclusion that coregulated genes are colocalizing in space, which may be true, but impossible to assert since this colocalization would mostly simply reflect the distance separating them from their respective centromeres. Similar precautions apply when studying the colocalization of regions positioned along the same chromosome, which will mechanically present enriched contacts compared to the average contacts over the entire genome, if the distance separating them not included in the null model. 


\section{Aknowledgements}

This research was supported by funding to R.K. from the European Research Council under the 7th Framework Program (FP7/2007-2013) / ERC grant agreement 260822. M.M. is the recipient of an Association pour la Recherche sur le Cancer fellowship 20100600373.

\section{References}

1. Z. Duan, M. Andronescu, K. Schutz, et al. (2010) A three-dimensional model of the yeast genome, Nature. 465, 363-367.

2. J. Dekker, K. Rippe, M. Dekker, et al. (2002) Capturing Chromosome Conformation, Science. 295, 1306-1311.

3. T. Sexton, E. Yaffe, E. Kenigsberg, et al. (2012) Three-Dimensional Folding and Functional Organization Principles of the Drosophila Genome, Cell. 148, 458-472.

4. H. Marie-Nelly, M. Marbouty, A. Cournac, et al. (2014) Filling annotation gaps in yeast genomes using genome-wide contact maps, Bioinformatics. btu 162.

5. E. Lieberman-Aiden, N.L. van Berkum, L. Williams, et al. (2009) Comprehensive Mapping of Long-Range Interactions Reveals Folding Principles of the Human Genome, Science. 326, 289-293.

6. W. de Laat and J. Dekker (2012) 3C-based technologies to study the shape of the genome, Methods. 58, 189-191.

7. M. Marbouty, A. Cournac, J.-F. Flot, et al. (2014) Metagenomic chromosome conformation capture (meta3C) unveils the diversity of chromosome organization in microorganisms, eLife. 3, e03318.

8. P. Oza, S.L. Jaspersen, A. Miele, et al. (2009) Mechanisms that regulate localization of a DNA double-strand break to the nuclear periphery, Genes \& Development. 23, 912-927.

9. J.M. O’Sullivan, S.M. Tan-Wong, A. Morillon, et al. (2004) Gene loops juxtapose promoters and terminators in yeast, Nature Genetics. 36, 1014-1018.

10. M.A. Mitchell and P.B. Dervan (1982) Interhelical DNA-DNA crosslinking. Bis(monoazidomethidium)octaoxahexacosanediamine: a probe of packaged nucleic acid, Journal of the American Chemical Society. 104, 4265-4266.

11. B.R. Lajoie, J. Dekker, and N. Kaplan The Hitchhiker's guide to Hi-C analysis: Practical guidelines, Methods.

12. A.A. Gavrilov, E.S. Gushchanskaya, O. Strelkova, et al. (2013) Disclosure of a structural milieu for the proximity ligation reveals the elusive nature of an active chromatin hub, Nucleic Acids Research.

13. T. Nagano, Y. Lubling, T.J. Stevens, et al. (2013) Single-cell Hi-C reveals cell-to-cell variability in chromosome structure, Nature. 502, 59-64.

14. H.J.G. van de Werken, G. Landan, S.J.B. Holwerda, et al. (2012) Robust 4C-seq data analysis to screen for regulatory DNA interactions, Nature Methods. 9, 969-972.

15. D.A. Thompson, S. Roy, M. Chan, et al. (2013) Evolutionary principles of modular gene 
regulation in yeasts, eLife. 2 ,

16. E. Yaffe and A. Tanay (2011) Probabilistic modeling of Hi-C contact maps eliminates systematic biases to characterize global chromosomal architecture, Nature Genetics. 43, $1059-1065$.

17. A. Cournac, H. Marie-Nelly, M. Marbouty, et al. (2012) Normalization of a chromosomal contact map, BMC Genomics. 13, 436.

18. M. Imakaev, G. Fudenberg, R.P. McCord, et al. (2012) Iterative correction of Hi-C data reveals hallmarks of chromosome organization, Nature Methods. 9, 999-1003.

19. B. Langmead and S.L. Salzberg (2012) Fast gapped-read alignment with Bowtie 2, Nature Methods. 9, 357-359.

20. H. Marie-Nelly, M. Marbouty, A. Cournac, et al. (2014) High-quality genome (re)assembly using chromosomal contact data, Nature Communications. 5,.

21. D.M. Witten and W.S. Noble (2012) On the assessment of statistical significance of threedimensional colocalization of sets of genomic elements, Nucleic Acids Research. 40, 3849-3855.

\section{Figure legends:}

Fig. 1. Photography of gel electrophoresis migration of DNA at various steps of a $3 C$ library

construction. (a) Non digested control (1); Digested control (2); 3C library (3). (b) Processing of a $3 \mathrm{C}$ library for Illumina sequencing. Profile after shearing (4); profile following reparation, addition of 3' A-tail and ligation of PE adapters (5); profile after size selection (400-800 bp) and PCR amplification (6).

Fig. 2. Distribution of the different types of molecules (green lines) sequenced from a 3C library, plotted as a function of the number of restriction sites (red bars) between the two pair-reads (black arrowheads). Sonicated sites are indicated with black twisted lines. The directionality of the reads according to the coordinates of the reference genome are indicated by + and - symbols and are used to characterize different sub-population in the library (see Cournac et al. 2012 for details). If the religation events were truly random between two RFs, each one of the four extremities of two restriction fragments would have 
the same probability to be ligated with each of the three others. However, because restriction efficiency is far from being $100 \%$ and because of the occurrence of other types of religation events (circularization, etc.), neighboring RFs present variations in the distribution of ligation events, with "uncuts" or "loops" events being overrepresented which do not reflect "contact" information but biochemical or physical biases. The grey bar indicates the number of restriction sites needed before all the different categories of events are equally represented within the population, corresponding to molecules that are retained for further analysis.

Fig. 3. (a) Raw genomic contact map of Saccharomyces cerevisiae, with each vector of the matrix representing 10 restriction fragments. (b) The same matrix after SCN normalization. (c) Pearson correlation representation of the normalized matrix.

Fig. 4. Illustration of the influence of crosslink concentration on the genomewide contact profile. S. cerevisiae contact maps obtained as described in this methods, but with varying formaldehyde concentrations ( $1 \%, 2 \%$ and $3 \%)$. Each contact map contains approximately the same amount of PE reads. 
Fig. 1

A

$6 \mathrm{~kb}$

$1 \mathrm{~kb}$
B

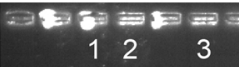

$1000 \mathrm{bp}$

$500 \mathrm{bp}$

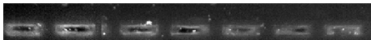

$4-5 \quad 6$

$-$ 
Fig. 2

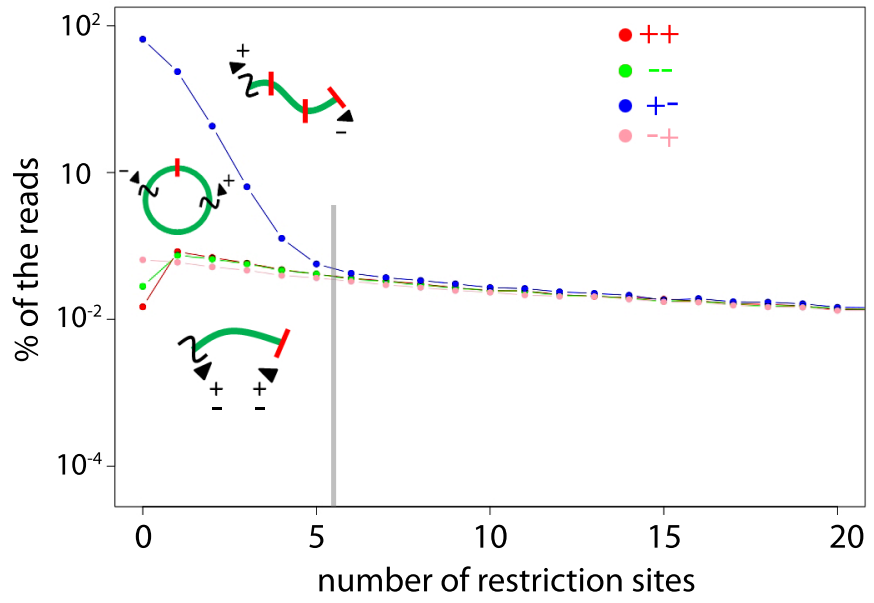




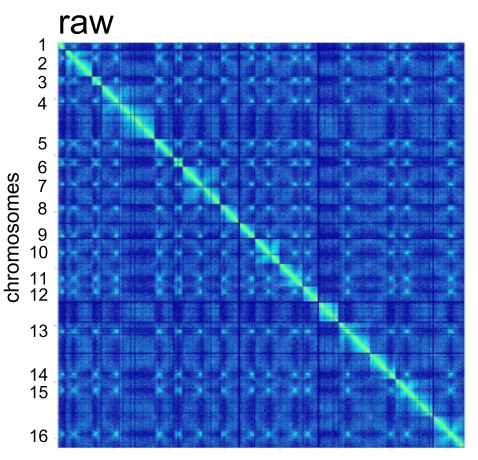

Fig. 3

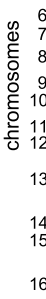

raw

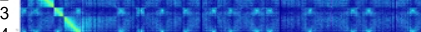

13
14
5
16 normalized

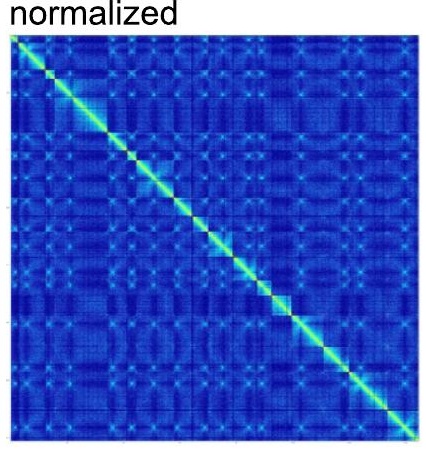

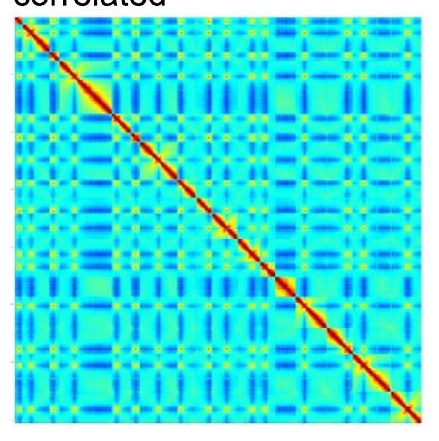

\section{correlated}

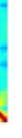

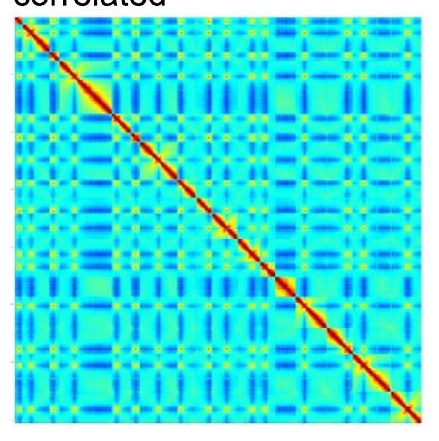


